\title{
An Approximately MAI-Free Multiaccess OFDM System in Fast Time-Varying Channels
}

\author{
Layla Tadjpour, Student Member, IEEE, Shang-Ho Tsai, Member, IEEE, and C.-C. Jay Kuo, Fellow, IEEE
}

\begin{abstract}
A new multiuser orthogonal frequency division multiplexing (OFDM) transceiver with preceding, called the precoded multiuser OFDM (PMU-OFDM) system, was recently introduced by Tsai et al. (2005). The PMU-OFDM system can reduce multiaccess interference (MAI) due to the carrier frequency offset (CFO) to a negligible amount by preceding the data of each user with a codeword selected from either even or odd Hadamard-Walsh codes. The performance of the PMU-OFDM system in a mobile environment, where the channel response varies within one OFDM symbol due to the Doppler effect, is evaluated in this paper. It is shown by analysis and simulation that the use of even or odd Hadamard-Walsh codewords can greatly reduce the MAI effect due to the Doppler effect as well. Generally, all Hadamard-Walsh codewords except for the all-one codeword result in considerable suppression of intercarrier interference (ICI) induced by the Doppler effect. Furthermore, we discuss codeword priority schemes to minimize ICI as much as possible. Finally, simulations results are given to demonstrate the advantage of PMU-OFDM over OFDMA with a codeword priority scheme in the Doppler environment. We show that the PMU-OFDM system with odd Hadamard-Walsh codewords outperforms a half-loaded OFDMA system in a mobile environment.
\end{abstract}

Index Terms-Carrier frequency offset (CFO), Doppler effect, fast fading, multiaccess interference (MAI), multiuser orthogonal frequency division multiplexing (OFDM), precoding, precoded multiuser OFDM (PMU-OFDM), time varying.

\section{INTRODUCTION}

O RTHOGONAL frequency division multiplexing (OFDM) provides a promising technique for high data rate transmission applications, since an OFDM system can combat the intersymbol interference (ISI) effect due to frequency-selective fading with a simple transceiver structure [2], [4]. OFDM has been used in wireless applications such as wireless local

Manuscript received May 25, 2006; revised October 12, 2006. The associate editor coordinating the review of this manuscript and approving it for publication was Prof. Brian L. Evans. This work was supported in part by the Integrated Media Systems Center, a National Science Foundation Engineering Research Center, Cooperative Agreement No. EEG-9529152. This work was presented in part at the 2005 Asilomar Conference on Signals, Systems and Computers, Pacific Grove, CA. Any opinions, findings, and conclusions or recommendations expressed in this material are those of the authors and do not necessarily reflect those of the National Science Foundation.

L. Tadjpour and C.-C. J. Kuo are with the Department of Electrical Engineering and Integrated Media Systems Center, University of Southern California, Los Angeles, CA 90089-2564 USA (e-mail: tadjpour@usc.edu; cckuo@sipi.usc.edu).

S.-H. Tsai is with the Department of Electrical and Control Engineering, National Chiao Tung University, Hsinchu 300, Taiwan (e-mail: shanghot@mail. nctu.edu.tw).

Color versions of one or more of the figures in this paper are available online at http://ieeexplore.ieee.org.

Digital Object Identifier 10.1109/TSP.2007.894260 area network standards (i.e., the IEEE 802.11 family) and the metropolitan area network standards (i.e., the IEEE 802.16 family). OFDM converts a frequency-selective fading channel into $N$ parallel flat fading channels by transmitting a block of $N$ information symbols in parallel on $N$ subcarriers. Through a careful design, a broadband channel can be efficiently shared by overlapping subchannels that have the minimum frequency separation required to maintain orthogonality while the fading effect can be mitigated by $N$ one-tap equalizers in the receiver. To mitigate effects of ISI and intercarrier interference (ICI) caused by the delay spread, each OFDM block is preceded by a cyclic prefix $(\mathrm{CP})$ whose length is at least equal to the channel length.

Historically, OFDM has been designed for applications with little mobility. However, mobile OFDM technology has recently attracted a lot of attention for three reasons. First, it is desirable to provide high-quality broadband services in a mobile environment when we consider the next generation wireless communication system. Second, emerging wireless communication systems are expected to lie in higher spectral bands so that they are more sensitive to the physical movement of users and their surroundings. Third, more subchannels are needed to enhance OFDM bandwidth efficiency, which implies the use of a longer OFDM block. Then, the effective channel variation rate over one OFDM block increases.

Multiuser OFDM systems have been developed to meet the need of wireless multiaccess, including time division multiaccess OFDM (TDMA-OFDM), multicarrier code division multiple access (MC-CDMA) [8], and orthogonal frequency division multiple access (OFDMA) [10]. MC-CDMA inherits the ISI-resilient property from OFDM. However, its capacity is limited by multiaccess interference (MAI) in frequency-selective fading channels. OFDMA has been adopted by the IEEE 802.16e standard, also known as WiMax. In OFDMA, a subset of subcarriers is assigned to each user. The division of subcarriers among users is usually called frequency division multiple access (FDMA) [12]. To mitigate the frequency-selective fading effect for each user, subcarriers assigned to a user can be spread across the spectrum [12]. OFDMA is MAI-free when time and frequency are perfectly synchronized. However, it is sensitive to the carrier frequency offset (CFO) and the Doppler spread, which are common in time-varying channels. The mismatch of oscillators in the transceiver or the rapid variation of the channel over one OFDM symbol destroys the orthogonality among subcarriers, and results in ICI and MAI in multiuser OFDM systems.

Since ICI degrades the performance of OFDM systems, its suppression has been intensively studied. Time domain 


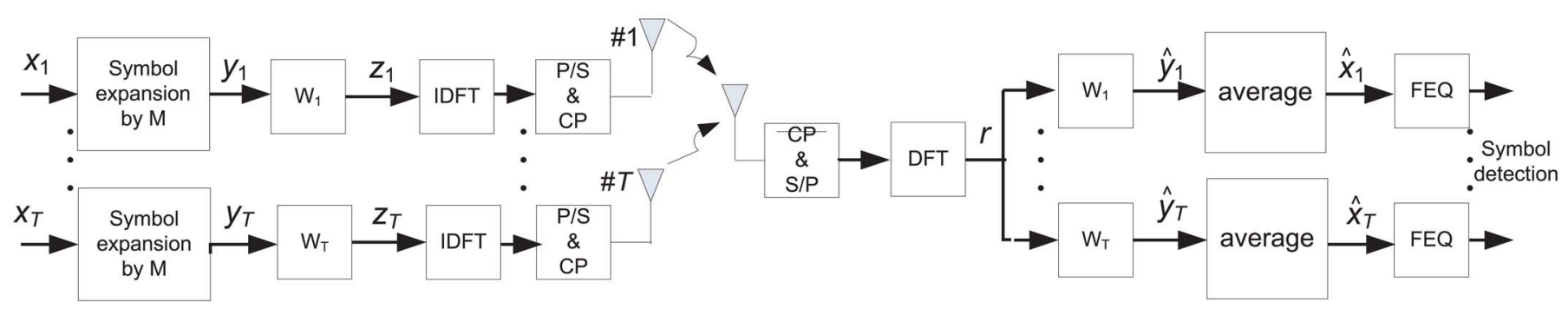

Fig. 1 Block diagram of the PMU-OFDM system.

equalizers designed based on the criterion of minimum mean squared errors (MMSE) were proposed to mitigate ICI in [15]. Lower complexity MMSE-based equalization techniques were considered in [3] and [17]. In [18] and [23], each data symbol is mapped into a group of subcarriers with predefined coefficients so that ICI among subcarriers within each group can be cancelled.

Furthermore, a time-varying channel during an OFDM block of an user degrades the performance of this user and induces MAI for others. There is little work on MAI suppression caused by the Doppler spread in MC-CDMA or OFDMA. Without efficient MAI suppression in a mobile environment, one may resort to a more complicated multiuser detection (MUD) technique for symbol detection, whose computational complexity increases exponentially with the number of active users [22]. Thus, a multiuser OFDM system that is robust to the Doppler spread effect is very desirable.

A new multiuser OFDM transceiver, called the precoded multiuser OFDM (PMU-OFDM), was introduced and analyzed in [19]. The PMU-OFDM system has three interesting properties. First, when the number of parallel transmit symbols $N$ is sufficiently large, it is approximately MAI-free. Second, PMUOFDM is robust to time asynchronism. Hence, low-complexity Hadamard-Walsh codes can be used in uplink transmission. Third, PMU-OFDM is approximately MAI-free in the presence of CFO due to the mismatch of oscillators in the transceiver using even or odd Hadamard-Walsh codewords.

In this paper, we will investigate the performance of PMU-OFDM in the presence of time-varying channels. We will prove that PMU-OFDM reduces MAI due to the Doppler spread to a negligible amount with even or odd Hadamard-Walsh codewords. We will also show that PMU-OFDM employing a subset of the Hadamard-Walsh codewords with many sign changes has self-ICI cancellation in a Doppler environment. We will compare PMU-OFDM with OFDMA, and demonstrate that PMU-OFDM with odd Hadamard-Walsh codewords outperforms half-loaded OFDMA in a Doppler environment.

The rest of this paper is organized as follows. The PMU-OFDM system and the Rayleigh time-varying channel model are reviewed in Section II. We analyze the MAI effect of PMU-OFDM due to the Doppler effect and show how to achieve the approximately MAI-free property in Section III, where the ICI effect resulting from the Doppler spread is also discussed. We also discuss the codeword priority scheme for PMU-OFDM and show that users using Hadamard-Walsh codewords with a higher sign change number experience less
ICI. The Monte Carlo simulation results are presented in Section IV and performance comparison between PMU-OFDM and OFDMA is conducted. Finally, concluding remarks are given in Section V.

\section{SYSTEM MODEL}

In this section, we provide a brief review to the PMU-OFDM system [19] whose block diagram is shown in Fig. 1. The input of user $j$ is an $N \times 1$ vector $\mathbf{x}_{j}$. Each symbol in $\mathbf{x}_{j}$ is repeated by $M$ times to form an $N M \times 1$ vector $\mathbf{y}_{j}$ as

$y_{j}[v+k M]=x_{j}[k], \quad 0 \leq k \leq N-1 ; \quad 0 \leq v \leq M-1$.

In the next stage, $\mathbf{y}_{j}$ is multiplied by a diagonal matrix $\mathbf{W}_{j}$ of size $N M \times N M$ whose diagonal elements are obtained by repeating the $j$ th column of an $M \times M$ real Hadamard matrix D by $N$ times, i.e.,

$$
\mathbf{W}_{j}=\operatorname{diag}\left(\mathbf{d}_{j}^{t}, \mathbf{d}_{j}^{t} \ldots \mathbf{d}_{j}^{t}\right)
$$

where $\mathbf{d}_{j}^{t}$ is the transpose of the $j$ th column of $\mathbf{D}$. Since $\mathbf{D}$ is an orthogonal matrix, the following property holds:

$$
\sum_{v=0}^{M-1} w_{i}[v+k M] w_{j}[v+k M]= \begin{cases}M, & i=j \\ 0, & i \neq j\end{cases}
$$

where $w_{j}[v+k M]$ is the $v+k M$ th diagonal element of diagonal matrix $\mathbf{W}_{\mathbf{j}}, 0 \leq v \leq M-1$ and $0 \leq k \leq N-1$. After passing through $\mathbf{W}_{j}$, the resultant $N M \times 1$ vector $\mathbf{z}_{j}$ can be written as

$$
z_{j}[l]=w_{j}[l] y_{j}[l], \quad 0 \leq l \leq N M-1
$$

where $l=v+k M$ is the frequency index. Next, each coded vector is passed through the $N M$-point inverse DFT (IDFT) matrix. Then, following the parallel-to-serial conversion, $N_{p} \mathrm{CP}$ are inserted at the beginning of each OFDM symbol. The $j$ th transmitted signal over a block is thus given by

$s_{j}(n)=\frac{1}{N M} \sum_{\Omega=0}^{N M-1} w_{j}[\Omega] y_{j}[\Omega] e^{j \frac{2 \pi}{N M} \Omega n}, \quad-N_{p} \leq n \leq N M$.

These symbols are fed to the multiple access channel. We consider the uplink scenario where each user experiences a different 
channel but under the assumption of synchronous communication. The time-varying channel impulse response for any user can be expressed by [16]

$$
h(n ; \tau)=\sum_{d=0}^{N_{h}-1} g(n ; d) \delta(\tau-d)
$$

where $n$ and $\tau$ denote time and delay, respectively, $N_{h}$ is the maximum length of channel impulse response, $g(n ; d)$ is the channel coefficient gain at time $n$ for the $d$ th tap, and $\delta(\cdot)$ is the Kronecker delta function. Coefficient $g(n ; d)$ is assumed to be a complex Gaussian random variable with zero mean and unit variance. Usually, $g(n ; d)$ is equal to $\beta_{d} e^{-j 2 \pi \Phi_{d}(n)}$, where $\beta_{d}$ and $\Phi_{d}(n)$ are random variables with the Rayleigh and the uniform distributions, respectively. Note that $\Phi_{d}(n)$ is the Doppler frequency for every channel path $d$ and it is the only time-varying factor in this model. The Doppler spread effect is caused by the difference in Doppler frequencies $\Phi_{d}(n)$ of different channel paths. If $\Phi_{d}(n)$ is the same for all channel paths, we have only the Doppler shift but not the Doppler spread. The latter is often used to model a time-invariant channel with CFO.

Also, the typical wide-sense stationary uncorrelated scattering (WSSUS) channel model is adopted [11], [16]. Then, we have

$$
E\left[g(n ; d) g^{*}\left(m ; d^{\prime}\right)\right]=\phi(n-m) \sigma_{d}^{2} \delta\left(d-d^{\prime}\right)
$$

where $\sigma_{d}^{2}$ is the variance of the $d$ th tap and $\phi(n-m)$ is the time-autocorrelation function. One classic time-autocorrelation function is the Jakes model [11], which can be written as

$$
\phi(n-m)=J_{0}\left(2 \pi f_{D} T_{s}(n-m)\right)
$$

where $J_{0}(x)$ is the zeroth-order Bessel function of the first kind, $f_{D}$ is the maximum Doppler frequency, and $T_{s}$ is the sampling rate. The Jakes model is adopted for the time-correlation function throughout this paper. If the $\mathrm{CP}$ duration is chosen such that $N_{p} \geq N_{h}$, the received signal after removing the cyclic prefix is given by

$$
r(n)=\sum_{i=1}^{T} \sum_{d=0}^{N_{h}-1} g_{i}(n ; d) s_{i}(n-d)+e(n)
$$

where $e(n)$ is the discrete-time additive white Gaussian noise, $g_{i}(n ; d)$ is the channel complex coefficient of user $i$, and $T$ is the number of multiple access users. Next, each block of size $N M$ is converted from serial to parallel and then passed through the DFT matrix. The output of DFT can be expressed as

$$
r[l]=\sum_{n=0}^{N M-1} r(n) e^{-j \frac{2 \pi}{N M} n l}, \quad 0 \leq l \leq N M-1 .
$$

To detect the symbol transmitted by the $j$ th user, the $N M \times$ 1 output vector of DFT is multiplied by $\mathbf{W}_{j}$ and the resultant output, denoted by $\hat{\mathbf{y}}_{j}$, is averaged over $M$ symbols such that

$$
\begin{aligned}
\hat{x}_{j}[k] & =\frac{1}{M} \sum_{v=0}^{M-1} \hat{y}_{j}[v+k M] \\
& =\frac{1}{M} \sum_{v=0}^{M-1} r[v+k M] w_{j}[v+k M]
\end{aligned}
$$

where $0 \leq k \leq N-1$. By substituting (5), (9), and (10) into (11), we have

$$
\begin{aligned}
\hat{x}_{j}[k]= & \frac{1}{M} \sum_{v=0}^{M-1} w_{j}[v+k M] \frac{1}{N M} \\
& \times \sum_{i=1}^{T} \sum_{n, \Omega=0}^{N M-1} y_{i}[\Omega] w_{i}[\Omega] \sum_{d=0}^{N_{h}-1} g_{i}(n ; d) \\
& \cdot\left\{e^{j \frac{2 \pi}{N M} \Omega(n-d)} e^{-j \frac{2 \pi}{N M} n(v+k M)}\right\}+\hat{e}[k]
\end{aligned}
$$

where

$$
\hat{e}[k]=\frac{1}{M} \sum_{v=0}^{M-1} e[v+k M] w_{j}[v+k M]
$$

and

$$
e[v+k M]=\sum_{n=0}^{N M-1} e(n) e^{-j \frac{2 \pi}{N M} n(v+k M)} .
$$

By (1), we get

$$
y_{i}[\Omega]=y_{i}[u+f M]=x_{i}[f]
$$

where $\Omega=u+f M$, with $0 \leq u \leq M-1$ and $0 \leq f \leq$ $N-1$. Since all symbols of an individual user use the same Hadamard-Walsh code, we have $w_{i}[u+f M]=w_{i}[u]$ and $w_{j}[v+k M]=w_{j}[v]$. By defining

$$
\zeta_{i, j}(n, d)=\frac{1}{M} \sum_{v=0}^{M-1} \sum_{u=0}^{M-1} w_{i}[u] w_{j}[v] e^{-j \frac{2 \pi}{N M}(v-u) n} e^{-j \frac{2 \pi}{N M} u d}
$$

and using (15), we can rewrite (12) as

$$
\begin{array}{r}
\hat{x}_{j}[k]=\sum_{i=1}^{T} \sum_{f=0}^{N-1} x_{i}[f] \frac{1}{N M} \sum_{n=0}^{N M-1} \sum_{d=0}^{N_{h}-1}\left[g_{i}(n ; d) \zeta_{i, j}(n, d)\right. \\
\left.\times e^{-j \frac{2 \pi}{N M}(f M) d} e^{-j \frac{2 \pi}{N}(k-f) n}\right]+\hat{e}[k] .
\end{array}
$$

Finally, to mitigate the fading effect on received symbols, $\hat{x}_{j}[k]$ is passed through a frequency equalizer (FEQ), which will be explained later.

Even though PMU-OFDM may appear to be similar to MC-CDMA in the system diagram, we should emphasize that the summation in the PMU-OFDM receiver is preformed before the gain multiplication, which is different from that of MC-CDMA systems. This difference will lead to different performance in the multipath fading channel or a CFO environment [19]. For more detailed performance comparison between MC-CDMA and PMU-OFDM, we refer to [19].

\section{ANALYSIS OF PMU-OFDM UNDER THE DOPPLER EFFECT}

The overall Doppler effect in a mobile environment can be divided into two parts. One is the interference from symbols of other users due to the time-varying channel while the other is the interference from neighboring subcarriers (i.e., Doppler ICI) of the same user to result in symbol distortions. They are called the Doppler MAI effect and the self-Doppler effect, respectively. We analyze the performance of PMU-OFDM, and derive its average MAI and ICI expressions in this section. 


\section{A. Analysis of Doppler MAI}

From (17), the MAI to the $k$ th symbol of user $j$ due to user $i$, denoted by $\mathrm{MAI}_{j \leftarrow i}[k]$, is given by

$$
\begin{aligned}
\operatorname{MAI}_{j \leftarrow i}[k]= & \sum_{f=0}^{N-1} x_{i}[f] \frac{1}{N M} \sum_{n=0}^{N M-1} \sum_{d=0}^{N_{h}-1} g_{i}(n ; d) \\
& \times \zeta_{i, j}(n, d) e^{-j \frac{2 \pi}{N M}(f M) d} e^{-j \frac{2 \pi}{N}(k-f) n} .
\end{aligned}
$$

The averaged $\mathrm{MAI}_{j \leftarrow i}[k]$ power is $E\left\{\left|\mathrm{MAI}_{j \leftarrow i}[k]\right|^{2}\right\}$. Let

$$
\zeta_{i, j}(n, d)=\eta_{i, j}(n) e^{-j \frac{2 \pi}{N M} u d}
$$

where

$$
\eta_{i, j}(n)=\frac{1}{M} \sum_{u, v=0}^{M-1} w_{i}[u] w_{j}[v] e^{-j \frac{2 \pi}{N M}(v-u) n} .
$$

Assuming that $x_{i}[f]$ and $g_{i}(n ; d)$ are uncorrelated, we can get from (18) that

$$
\begin{aligned}
E\left\{\left|\mathrm{MAI}_{j \leftarrow i}[k]\right|^{2}\right\} & \left(\frac{1}{N M}\right)^{2} \sum_{f, f^{\prime}=0}^{N-1} E\left\{x_{i}[f] x_{i}^{*}\left[f^{\prime}\right]\right\} \sum_{n, m=0}^{N M-1} \sum_{d, d^{\prime}=0}^{N_{h}-1} \\
& \cdot\left\{E\left\{g_{i}(n ; d) g_{i}^{*}\left(m ; d^{\prime}\right)\right\} \eta_{i, j}(n) \eta_{i, j}^{*}(m)\right. \\
& \left.\times e^{-j \frac{2 \pi}{N M}(u+f M)\left(d-d^{\prime}\right)} e^{-j \frac{2 \pi}{N}(k-f)(n-m)}\right\} .
\end{aligned}
$$

It is also assumed that cross correlation between $x_{i}[f]$ is zero; i.e., $E\left\{x_{i}[f] x_{i}^{*}\left[f^{\prime}\right]\right\}=\sigma_{x_{i}}^{2} \delta\left(f-f^{\prime}\right)$. Then, by (7) and (8), the averaged power of $\mathrm{MAI}_{j \leftarrow i}[k]$ can be found as

$$
\begin{aligned}
E\left\{\left|\mathrm{MAI}_{j \leftarrow i}[k]\right|^{2}\right\} & \\
= & N_{h}\left(\frac{\sigma_{d} \sigma_{x_{i}}}{N M}\right)^{2} \sum_{f=0}^{N-1} \sum_{n, m=0}^{N M-1}\left\{J_{0}\left(2 \pi f_{D} T_{s}(n-m)\right)\right. \\
& \left.\cdot \eta_{i, j}(n) \eta_{i, j}^{*}(m) e^{-j \frac{2 \pi}{N}(k-f)(n-m)}\right\}
\end{aligned}
$$

Consider the case $f=k$, where the resulting MAI is denoted by $\operatorname{MAI}_{j \leftarrow i}^{(0)}[k]$. Then, from $(21)$, the average power of $\operatorname{MAI}_{j \leftarrow i}^{(0)}[k]$ is given by

$$
\begin{aligned}
E\left\{\left|\mathrm{MAI}_{j \leftarrow i}^{(0)}[k]\right|^{2}\right\} & =N_{h}\left(\frac{\sigma_{d} \sigma_{x_{i}}}{N M}\right)^{2} \\
& \times \sum_{n, m=0}^{N M-1} J_{0}\left(2 \pi f_{D} T_{s}(n-m)\right) \eta_{i, j}(n) \eta_{i, j}^{*}(m) .
\end{aligned}
$$

It is known [9] that $M$ Hadamard-Walsh codewords can be divided into two groups of $M / 2$ codewords. One is the set of even codewords satisfying

$$
w_{i}[a]=w_{i}[M-1-a], \quad 0 \leq a \leq M / 2-1,
$$

and the other is the set of odd codewords given by

$$
w_{i}[a]=-w_{i}[M-1-a], \quad 0 \leq a \leq M / 2-1 .
$$

In the following, we will prove that if only $M / 2$ even or odd codewords of $M$ Hadamard-Walsh codewords are used,
$\operatorname{MAI}_{j \leftarrow i}^{(0)}[k]$ can be reduced to a negligible amount. Then, we observe that $\mathrm{MAI}_{j \leftarrow i}^{(0)}[k]$ is the dominating MAI by computer simulation in Section IV. Thus, by reducing $\mathrm{MAI}_{j \leftarrow i}^{(0)}[k]$ to a negligible amount, the total MAI is also reduced substantially.

Theorem: Suppose that only the $M / 2$ even or the $M / 2$ odd codewords of $M$ Hadamard-Walsh codewords are used, $\operatorname{MAI}_{j \leftarrow i}^{(0)}[k]$ is approximately zero if the normalized Doppler frequency is less than $1 /(2 \pi N M)$.

Proof: It can be easily shown that $\sum_{p=1}^{M-1} \sum_{q=0}^{M-1-p} w_{i}[p+$ $q] w_{j}[q]=0, i \neq j$, (also, see [21]). Then, we can rewrite (22) such that it includes this term. To do so, we can use the following:

$$
\begin{aligned}
& \sum_{u=0, u \neq v}^{M-1} \sum_{v=0}^{M-1} \alpha(u-v) w_{i}[u] w_{j}[v] \\
& =\sum_{p=1}^{M-1}\left\{\alpha(p) \sum_{q=0}^{M-1-p} w_{i}[p+q] w_{j}[q]\right. \\
& \left.+\alpha(-p) \sum_{q=0}^{M-1-p} w_{i}[q] w_{j}[p+q]\right\}
\end{aligned}
$$

where $\alpha(\cdot)$ can be any function. Note that if $i \neq j$, we have

$$
\begin{aligned}
\sum_{u=0}^{M-1} \sum_{v=0}^{M-1} \alpha(u-v) & w_{i}[u] w_{j}[v] \\
& =\sum_{u=0, u \neq v}^{M-1} \sum_{v=0}^{M-1} \alpha(u-v) w_{i}[u] w_{j}[v] .
\end{aligned}
$$

Also, even and odd codewords have the following property [19]:

$$
\sum_{q=0}^{M-1-p} w_{i}[p+q] w_{j}[q]=\sum_{q=0}^{M-1-p} w_{i}[q] w_{j}[p+q]
$$

Let $\alpha(u-v)=e^{-j 2 \pi / N M(v-u) n}$. Using (25)-(27), for $i \neq j$, $\eta_{i, j}(n)$ can be written as

$$
\eta_{i, j}(n)=\frac{2}{M} \sum_{p=1}^{M-1} \sum_{q=0}^{M-1-p} w_{i}[p+q] w_{j}[q] \cos \left(\frac{2 \pi}{N M} p n\right) .
$$

By substituting (28) in (22) and making some rearrangement, we can obtain the average power of $\mathrm{MAI}_{j \leftarrow i}^{(0)}[k]$ as

$$
\begin{aligned}
E\left\{\left|\mathrm{MAI}_{j \leftarrow i}^{(0)}[k]\right|^{2}\right\} \\
=N_{h}\left(\frac{2 \sigma_{d} \sigma_{x_{i}}}{N M^{2}}\right)^{2} \sum_{p=1}^{M-1} \sum_{q=0}^{M-1-p} w_{i}[p+q] w_{j}[q] \\
\quad \times \sum_{r=1}^{M-1} \sum_{s=0}^{M-1-r} w_{i}[r+s] w_{j}[s] \rho(p, r)
\end{aligned}
$$

where

$$
\begin{aligned}
\rho(p, r)=\sum_{n, m=0}^{N M-1} J_{0}\left(2 \pi f_{D} T_{s}(n-m)\right) \\
\quad \times \cos \left(\frac{2 \pi}{N M} p n\right) \cos \left(\frac{2 \pi}{N M} r m\right) .
\end{aligned}
$$




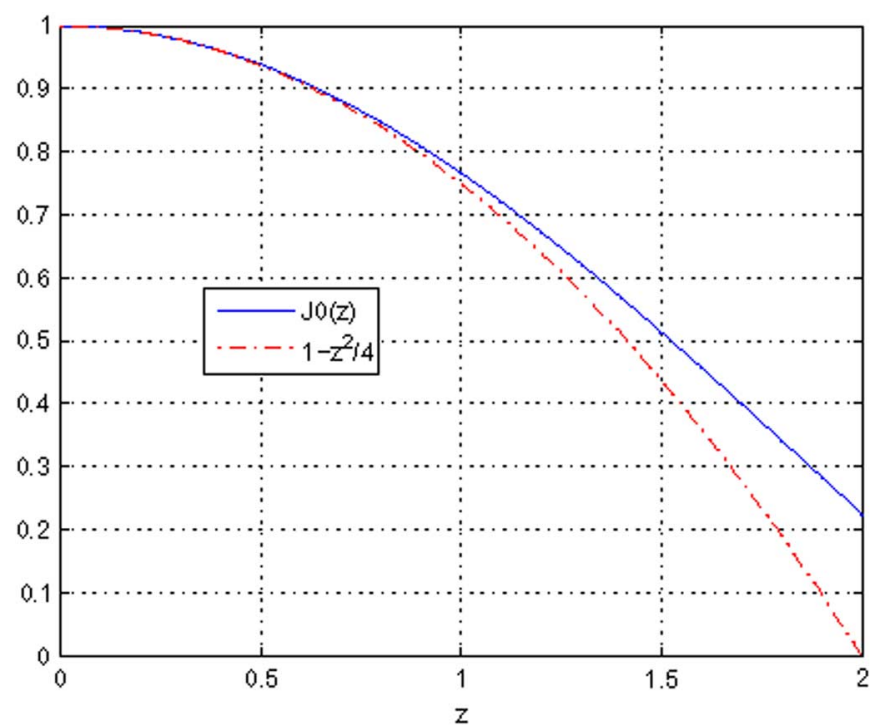

Fig. 2 Approximation of $J_{0}(z)$ by $1-\left((1 / 4) z^{2}\right) /\left((1 !)^{2}\right)$ for $z<1$.

Now, we need to show that $\rho(p, r)$ is a constant function so that $E\left\{\left|\mathrm{MAI}_{j \leftarrow i}^{(0)}[k]\right|^{2}\right\} \simeq 0$, for $f_{D} T_{s}<1 / 2 \pi N M$.

The zeroth-order Bessel function of the first kind can be expanded by the following power series [1]:

$$
J_{0}(z)=1-\frac{\frac{1}{4} z^{2}}{(1 !)^{2}}+\frac{\left(\frac{1}{4} z^{2}\right)^{2}}{(2 !)^{2}}-\frac{\left(\frac{1}{4} z^{2}\right)^{3}}{(3 !)^{2}}+\cdots
$$

As shown in Fig. 2, we can approximate $J_{0}(z)$ for $0<z<1$ by $1-(1 / 4) z^{2} /(1 !)^{2}$. By substituting this approximation for $J_{0}\left(2 \pi f_{D} T_{s}(n-m)\right)$ with $0 \leq n-m \leq N M, 0<f_{D} T_{s}<$ $1 / 2 \pi N M$ (or, equivalently, $0<2 \pi f_{D} \bar{T}_{s}(n-m)<1$ ), we obtain

$$
J_{0}\left(2 \pi f_{D} T_{s}(n-m)\right) \simeq\left(1-\pi^{2}\left(f_{D} T_{s}\right)^{2}(n-m)^{2}\right) .
$$

where $f_{D} T_{s}<(1 /(2 \pi N M))$. Based on the previous approximation, we can express $\rho(p, r)$ in (29) as

$$
\begin{aligned}
\rho(p, r) \simeq & \sum_{n=0}^{N M-1} \sum_{m=0}^{N M-1} \cos \left(\frac{2 \pi}{N M} p n\right) \cos \left(\frac{2 \pi}{N M} r m\right) \\
& -\pi^{2}\left(f_{D} T_{s}\right)^{2} \sum_{n=0}^{N M-1} n^{2} \cos \left(\frac{2 \pi}{N M} p n\right) \\
& \times \sum_{m=0}^{N M-1} \cos \left(\frac{2 \pi}{N M} r m\right) \\
& -\pi^{2}\left(f_{D} T_{s}\right)^{2} \sum_{n=0}^{N M-1} \cos \left(\frac{2 \pi}{N M} p n\right) \\
& \times \sum_{m=0}^{N M-1} m^{2} \cos \left(\frac{2 \pi}{N M} r m\right) \\
& +2 \pi^{2}\left(f_{D} T_{s}\right)^{2} \sum_{n=0}^{N M-1} n \cos \left(\frac{2 \pi}{N M} p n\right) \\
& \times \sum_{m=0}^{N M-1} m \cos \left(\frac{2 \pi}{N M} r m\right) .
\end{aligned}
$$

It can easily be shown that $\sum_{n=0}^{N M-1} \cos ((2 \pi) /(N M) p n)=$ $\sum_{m=0}^{N M-1} \cos ((2 \pi) /(N M) r m)=0$. Also, we know from [7] that

$$
\sum_{k=0}^{n-1} k \cos (k x)=\frac{n \sin \left(\frac{2 n-1}{2} x\right)}{2 \sin \left(\frac{x}{2}\right)}-\frac{1-\cos (n x)}{4 \sin ^{2}\left(\frac{x}{2}\right)}, \quad x \neq 0 .
$$

Therefore, for $p=1,2, \ldots M-1$ and $r=1,2, \ldots, M-1$, we get

$$
\begin{aligned}
& \sum_{m=0}^{N M-1} m \cos \left(\frac{2 \pi}{N M} r m\right) \\
& =\sum_{n=0}^{N M-1} n \cos \left(\frac{2 \pi}{N M} p n\right) \\
& =\frac{N M \sin \left(\frac{2 N M-1}{2} \frac{2 \pi}{N M} p\right)}{2 \sin \left(\frac{\pi}{N M} p\right)}-\frac{1-\cos (2 \pi p)}{4 \sin ^{2}\left(\frac{\pi}{N M} p\right)} \\
& =\frac{N M \sin \left(2 \pi p-\frac{p \pi}{N M}\right)}{2}=-\frac{N M}{\sin \left(\frac{p \pi}{N M}\right)} .
\end{aligned}
$$

By plugging (35) in (33)

$$
\rho(p, r) \simeq 2 \pi^{2}\left(f_{D} T_{s}\right)^{2}\left(\frac{N M}{2}\right)^{2}
$$

which is independent of $p$ and $r$. By substituting this approximate value of $\rho(p, r)$ back to (29), we obtain

$$
\begin{aligned}
E\left\{\left|\mathrm{MAI}_{j \leftarrow i}^{(0)}[k]\right|^{2}\right\} \simeq & 2 N_{h}\left(\frac{\pi f_{D} T_{s} \sigma_{d} \sigma_{x_{i}}}{M}\right)^{2} \\
& \times \sum_{p=1}^{M-1} \sum_{q=0}^{M-1-p} w_{i}[p+q] w_{j}[q] \\
& \times \sum_{r=1}^{M-1} \sum_{s=0}^{M-1-r} w_{i}[r+s] w_{j}[s] .
\end{aligned}
$$

One can show $\sum_{p=1}^{M-1} \sum_{q=0}^{M-1-p} w_{i}[p+q] w_{j}[q]=0$, if the even or odd Hadamard-Walsh codewords are used. For the detailed proof, we refer to [21]. Finally, we have

$$
E\left\{\left|\mathrm{MAI}_{j \leftarrow i}^{(0)}[k]\right|^{2}\right\} \simeq 0, \quad \text { if } f_{D} T_{s}<\frac{1}{2 \pi N M} .
$$

Equation (37) shows that PMU-OFDM is approximately MAI-free in a Doppler environment. This result is actually similar to that in a CFO environment [19]. We may consider a simple example for the illustration purpose. When $N=64$ and $M=16$, PMU-OFDM is approximately MAI-free when the normalized maximum Doppler frequency is less than $1.5 \times 10^{-4}$. For the carrier frequency $f_{c}=4 \mathrm{GHz}$ and the sampling frequency $F_{s}=1 / T_{s}=2 \mathrm{MHz}$, this maximum Doppler frequency is equivalent to a mobile speed of $81 \mathrm{~km} / \mathrm{h}$. Since $\mathrm{MAI}_{j \leftarrow i}^{(0)}[k]$ is the dominating MAI in a time-invariant CFO environment [19], the suppression of $\mathrm{MAI}_{j \leftarrow i}^{(0)}[k]$ reduces the total MAI due to CFO greatly. We will show by simulation in Section IV that $\operatorname{MAI}_{j \leftarrow i}^{(0)}[k]$ is the dominating MAI in a 
Doppler environment as well so that the total MAI can be suppressed significantly in a Doppler environment by suppressing $\mathrm{MAI}_{j \leftarrow i}^{(0)}[k]$ using odd or even Hadamard-Walsh codewords.

\section{B. Analysis of Doppler ICI and Symbol Distortion}

In this section, we investigate the impairment caused by user's self Doppler effect. Let $i=j$ in (17). The $k$ th detected symbol of user $j$ with no other active user is given by

$$
\begin{aligned}
\hat{x}_{j}[k]= & \sum_{f=0}^{N-1} x_{j}[f] \frac{1}{N M} \sum_{n=0}^{N M-1} \sum_{d=0}^{N_{h}-1} \\
& \times g_{j}(n ; d) \zeta_{j, j}(n, d) e^{-j \frac{2 \pi}{N M}(f M) d} \\
& \times e^{-j \frac{2 \pi}{N}(k-f) n}+\hat{e}[k] .
\end{aligned}
$$

We can rewrite (38) as

$$
\hat{x}_{j}[k]=x_{j}[k] H_{j}[k]+\mathrm{ICI}_{j}^{(0)}[k]+\mathrm{ICI}_{j}^{(1)}[k]+\hat{e}[k]
$$

where $H_{j}[k], \mathrm{ICI}_{j}^{(0)}[k]$, and $\mathrm{ICI}_{j}^{(1)}[k]$ are discussed later. The distortion factor $H_{j}[k]$ is obtained by putting $f=k$ and $u=v$ in $\zeta_{j, j}(n, d)$ in (38), i.e.,

$$
H_{j}[k]=\frac{1}{N M} \sum_{n=0}^{N M-1} \sum_{d=0}^{N_{h}-1} g_{j}(n ; d) \frac{1}{M} \sum_{v=0}^{M-1} e^{-j \frac{2 \pi}{N M}(v+k M) d}
$$

for $0 \leq v \leq M-1$, and $0 \leq d \leq N_{h}-1$. If $N M \gg v d$, then $e^{-j(2 \pi) /(N M) v d} \simeq 1,1 / M \sum_{v=0}^{M=1} e^{-j 2 \pi / N M v d} \simeq 1$. Note that since $0 \leq v \leq M-1$, and $0 \leq d \leq N_{h}-1, N M \gg v d$ is equivalent to $N \gg N_{h}-1$. Therefore, $H_{j}[k]$ can be written as

$$
H_{j}[k] \simeq \frac{1}{N M} \sum_{n=0}^{N M-1} \sum_{d=0}^{N_{h}-1} g_{j}(n ; d) e^{-j \frac{2 \pi}{N} k d} .
$$

That is, for every time index $n$ and any user $j$, we take the DFT of channel coefficient $g_{j}(n ; d)$ over path $d$ with frequency index $k$. The result is then averaged over one OFDM symbol to yield $H_{j}[k]$. When $N \gg N_{h}-1$, another interpretation of $H_{j}[k]$ is

$$
H_{j}[k] \simeq \sum_{d=0}^{N_{h}-1} g_{j}^{\mathrm{avg}}(d) e^{-j \frac{2 \pi}{N} k d}
$$

where $g_{j}^{\mathrm{avg}}(d)=(1) /(N M) \sum_{n=0}^{N M-1} g_{j}(n ; d)$ is the average of the $d$ th channel tap over one OFDM block. Therefore, $H_{j}[k]$ represents the DFT of $g_{j}^{\mathrm{avg}}(d)$. Often, subcarriers are much longer than the channel length and $H_{j}[k]$ can be computed from (42). In Section IV, we assume this condition is held by adopting $N=64$ and $N_{h}=4$ in Examples 3 and 4 .

We can mitigate the symbol distortion effect in the receiver using a frequency domain equalizer whose one tap gain for user $j$ is set to $\left(H_{j}[k]\right)^{-1}$. To this end, channel estimation for timevarying channel must be preformed. When the channel is timevarying within an OFDM block, the preamble-based training method may not work well. Periodic insertion of training symbols during transmission of every block has been suggested for OFDM in time-varying channels. It was shown in [5] that the best set of frequency domain pilot tones are those which are equally spaced. We adopt this technique to estimate the fast fading channel. Let $P$ be the number of equally spaced pilot tones at subchannels $\Lambda[k]=k \times N / P$, for $0 \leq k \leq P-1$. An estimate of $H_{j}[k]$ can be obtained at pilot tones via

$$
\hat{H}_{j}[\Lambda[k]]=\frac{\hat{x}_{j}[\Lambda[k]]}{x_{j}[\Lambda[k]]}+\frac{\left(\mathrm{ICI}_{j}^{(0)}[\Lambda[k]]+\mathrm{ICI}_{j}^{(1)}[\Lambda[k]]+\hat{e}[\Lambda[k]]\right)}{x_{j}[\Lambda[k]]} .
$$

Then, the estimate of $g_{j}^{\text {avg }}(d)$ is obtained through an IDFT of length $P$ as

$$
\hat{g}_{j}^{\mathrm{avg}}(d)=\frac{1}{P} \sum_{k=0}^{P-1} \hat{H}_{j}[\Lambda[k]] e^{\frac{j 2 \pi d k}{P}} .
$$

More sophisticated algorithms were suggested to improve the channel estimation performance in a Doppler environment. For example, two ICI mitigation techniques were proposed in [6] to improve the channel estimation performance in the presence of the Doppler effect. Other channel estimation methods for OFDM in time-varying channels were reported in [13] and [14].

The term $\mathrm{ICI}_{j}^{(0)}[k]$ in (39) is the interference from subcarriers with $f=k$ and $u \neq v$ to the desired subcarrier $f=k$; namely

$$
\begin{aligned}
\operatorname{ICI}_{j}^{(0)}[k]= & x_{j}[k] \frac{1}{N M} \sum_{n=0}^{N M-1} \sum_{d=0}^{N_{h}-1} g_{j}(n ; d)\left\{\left.\zeta_{j, j}(n, d)\right|_{u \neq v}\right\} \\
& \times e^{-j \frac{2 \pi}{N M}(k M) d} .
\end{aligned}
$$

Finally, the term $\mathrm{ICI}_{j}^{(1)}[k]$ in (39) is the sum of all interferences from subcarriers $f \neq k$, i.e.,

$$
\begin{aligned}
\mathrm{ICI}_{j}^{(1)}[k]= & \sum_{f=0, f \neq k}^{N-1} x_{j}[f] \frac{1}{N M} \sum_{n=0}^{N M-1} \sum_{d=0}^{N_{h}-1} g_{j}(n ; d) \zeta_{j, j}(n, d) \\
& \times e^{-j \frac{2 \pi}{N M}(f M) d} e^{-j \frac{2 \pi}{N}(k-f) n} .
\end{aligned}
$$

Since the ICI average power can be obtained in a manner similar to that in deriving the MAI average power, we omit the derivation and simply show the result. That is, the average power of $\mathrm{ICI}_{j}^{(0)}[k]$ is equal to

$$
\begin{aligned}
E\left\{\left|\mathrm{ICI}_{j}^{(0)}[k]\right|^{2}\right\}= & N_{h}\left(\frac{\sigma_{d} \sigma_{x_{j}}}{N M}\right)^{2} \sum_{n, m=0}^{N M-1} J_{0}\left(2 \pi f_{D} T_{s}(n-m)\right) \\
& \times\left\{\left.\eta_{j, j}(n)\right|_{u \neq v}\right\}\left\{\left.\eta_{j, j}^{*}(m)\right|_{u \neq v}\right\} .
\end{aligned}
$$

We can rewrite (25) for $i=j$ as

$$
\begin{aligned}
& \sum_{u, v=0, u \neq v}^{M-1} \alpha(u-v) w_{j}[u] w_{j}[v] \\
& =\sum_{p=1}^{M-1}\left\{\{\alpha(p)+\alpha(-p)\} \sum_{q=0}^{M-1-p} w_{j}[p+q] w_{j}[q]\right\} .
\end{aligned}
$$

Let $\alpha(u-v)=e^{-j(2 \pi) /(N M)(v-u) n}$. Then

$\left.\eta_{j, j}(n)\right|_{u \neq v}=\frac{2}{M} \sum_{p=1}^{M-1} \sum_{q=0}^{M-1-p} w_{j}[p+q] w_{j}[q] \cos \left(\frac{2 \pi}{N M} p n\right)$ 
By substituting (49) in (47) and using the approximate formula (32) for the Bessel function and (35), we have

$$
\begin{aligned}
E\left\{\left|\mathrm{ICI}_{j}^{(0)}[k]\right|^{2}\right\} \\
\simeq 2 N_{h}\left(\frac{\pi f_{D} T_{s} \sigma_{d} \sigma_{x_{j}}}{M}\right) \sum_{p=1}^{2} \sum_{q=0}^{M-1} w_{j}[p+q] w_{j}[q] \\
\quad \times \sum_{r=1}^{M-1} \sum_{s=0}^{M-1-r} w_{j}[r+s] w_{j}[s]
\end{aligned}
$$

The double summation term in (50) is found to be

$$
\begin{aligned}
& \sum_{p=1}^{M-1} \sum_{q=0}^{M-1-p} w_{j}[p+q] w_{j}[q] \\
&= \begin{cases}M(M-1) / 2, & \text { all-one codeword } \\
-M / 2, & \text { otherwise }\end{cases}
\end{aligned}
$$

since the left term of (51) is equal to

$\frac{1}{2} \sum_{u, v=0, u \neq v}^{M-1} w_{j}[u] w_{j}[v]=\frac{1}{2} \sum_{u=0}^{M-1} w_{j}[u] \sum_{v=0}^{M-1} w_{j}[v]-\frac{1}{2} \sum_{u=0}^{M-1} w_{j}^{2}[u]$.

In addition, $\sum_{u=0}^{M-1} w_{j}[u]$ is equal to $M$ for the all-one codeword and is 0 for all other codewords. From (50) and (51), $E\left\{\left|\mathrm{ICI}_{j}^{(0)}[k]\right|^{2}\right\} \simeq\left(N_{h}\right) /(2)\left(\pi f_{D} T_{s} \sigma_{d} \sigma_{x_{j}}\right)^{2}$, when the all-one codeword is not used and $f_{D} T_{s}<1 /(2 \pi N M)$. On the other hand, if the all-one codeword is used, we have $E\left\{\left|\mathrm{ICI}_{j}^{(0)}[k]\right|^{2}\right\} \simeq N_{h} / 2\left(\pi f_{D} T_{s} \sigma_{d} \sigma_{x_{j}}(M-1)\right)^{2}$. For instance, if $f_{D} T_{s}<1 /(2 \pi N M), E\left\{\left|\mathrm{ICI}_{j}^{(0)}[k]\right|^{2}\right\}<$ $N_{h} \sigma_{d}^{2} \sigma_{x_{j}}^{2} / 8 N^{2}$ for the all-one codeword and $E\left\{\left|\mathrm{ICI}_{j}^{(0)}[k]\right|^{2}\right\}<$ $\left(N_{h} \sigma_{d}^{2} \sigma_{x_{j}}^{2}\right) /\left(8 N^{2} M^{2}\right)$ for other codewords. by

We can also derive the average power of $\operatorname{ICI}_{j}^{(1)}[k]$, denoted

$$
\begin{aligned}
E\left\{\left|\mathrm{ICI}_{j}^{(1)}[k]\right|^{2}\right\}=N_{h} & \left(\frac{\sigma_{d} \sigma_{x_{j}}}{N M}\right)^{2} \sum_{f=0, f \neq k}^{N-1} \sum_{n, m=0}^{N M-1} \\
\times & \left\{J_{0}\left(2 \pi f_{D} T_{s}(n-m)\right) \eta_{j, j}(n)\right. \\
& \left.\times \eta_{j, j}^{*}(m) e^{-j \frac{2 \pi}{N}(k-f)(n-m)}\right\} .
\end{aligned}
$$

As compared with the average power of $\operatorname{ICI}_{j}^{(1)}[k]$, the average power of $\mathrm{ICI}_{j}^{(0)}[k]$ is negligible for all codewords for practical values of $N$ and $N_{h}$. This can be clearly explained by the following example.

Example: Let $N=64, N_{h}=4, M=16, f_{D} T_{s}=1 \times$ $10^{-4}$, and $\sigma_{d}^{2}=\sigma_{x_{j}}^{2}=1$. Then, $E\left\{\left|\mathrm{ICI}_{j}^{(0)}[k]\right|^{2}\right\} \simeq-40.5 \mathrm{~dB}$ for the all-one codeword, and $E\left\{\left|\mathrm{ICI}_{j}^{(0)}[k]\right|^{2}\right\} \simeq-64 \mathrm{~dB}$ for other codewords. Using (52) and the same set of parameters, we compute the value of $E\left\{\left|\mathrm{ICI}_{j}^{(1)}[k]\right|^{2}\right\}$ to be about $-10 \mathrm{~dB}$ for the user with the all-one codeword decibels, and between -25 and $-50 \mathrm{~dB}$ for all other users. Clearly, $E\left\{\left|\operatorname{ICI}_{j}^{(1)}[k]\right|^{2}\right\}$ is much larger than the corresponding values of $E\left\{\left|\mathrm{ICI}_{j}^{(0)}[k]\right|^{2}\right\}$.
Since $\operatorname{ICI}_{j}^{(1)}[k]$ is the dominant ICI, we only need to consider this ICI effect and ignore $\operatorname{ICI}_{j}^{(0)}[k]$. It will also be shown later in this section and in Section IV that the total interference from subcarriers $f \neq k$ of user $j$, i.e., $\operatorname{ICI}_{j}^{(1)}[k]$, with the all-one codeword is much larger than that of other codewords. It will also be shown in Section IV that the averaged ICI power depends on the assigned Hadamard-Walsh codeword.

\section{Codeword Priority Schemes for ICI Cancellation}

Since $\operatorname{ICI}_{j}^{(1)}[k]$ is the dominant ICI due to the Doppler spread effect, we would like to further investigate this term in this section. It is found that codewords with a higher number of sign changes tend to lead to a smaller ICI value. This can be explained as follows. Expression $\eta_{j, j}(n) \eta_{j, j}^{*}(m)$ in (52) can be divided into four terms: $\left\{\left.\eta_{j, j}(n)\right|_{u=v}\right\}\left\{\left.\eta_{j, j}^{*}(m)\right|_{u=v}\right\}$, $\left\{\left.\eta_{j, j}(n)\right|_{u=v}\right\}\left\{\left.\eta_{j, j}^{*}(m)\right|_{u \neq v}\right\}, \quad\left\{\left.\eta_{j, j}(n)\right|_{u \neq v}\right\}\left\{\left.\eta_{j, j}^{*}(m)\right|_{u=v}\right\}$, and $\left\{\left.\eta_{j, j}(n)\right|_{u \neq v}\right\}\left\{\left.\eta_{j, j}^{*}(m)\right|_{u \neq v}\right\}$.

Let $r_{j, j}[p]=\sum_{q=0}^{M-1-p} w_{j}[p+q] w_{j}[q], 1 \leq p \leq M-1$. Since $\left\{\left.\eta_{j, j}(n)\right|_{u=v}\right\}=1$, and by using (49), (52) can be written as

$$
\begin{aligned}
E\{\mid & \left.\left.\mathrm{ICI}_{j}^{(1)}[k]\right|^{2}\right\} \\
= & N_{h}\left(\frac{\sigma_{d} \sigma_{x_{j}}}{N M}\right)^{2} \sum_{f=0, f \neq k}^{N-1}\left\{\sum_{n, m=0}^{N M-1} J_{0}\left(2 \pi f_{D} T_{s}(n-m)\right)\right. \\
& \times e^{-j \frac{2 \pi}{N}(k-f)(n-m)} \\
& +\frac{2}{M} \sum_{p=1}^{M-1} r_{j, j}[p] \sum_{n, m=0}^{N M-1} J_{0}\left(2 \pi f_{D} T_{s}(n-m)\right) \\
& \times \cos \left(\frac{2 \pi}{N M} p n\right) e^{-j \frac{2 \pi}{N}(k-f)(n-m)} \\
& +\frac{2}{M} \sum_{p=1}^{M-1} r_{j, j}[p] \sum_{n, m=0}^{N M-1} J_{0}\left(2 \pi f_{D} T_{s}(n-m)\right) \\
& \times \cos \left(\frac{2 \pi}{N M} p m\right) e^{-j \frac{2 \pi}{N}(k-f)(n-m)} \\
& +\frac{4}{M^{2}}\left|\sum_{p=1}^{M-1} r_{j, j}[p]\right|^{2} \sum_{n, m=0}^{N M-1} J_{0}\left(2 \pi f_{D} T_{s}(n-m)\right) \\
& \left.\times \cos \left(\frac{2 \pi}{N M} p n\right) \cos \left(\frac{2 \pi}{N M} p m\right) e^{-j \frac{2 \pi}{N}(k-f)(n-m)}\right\} .
\end{aligned}
$$

Define

$$
\beta(p, k-f)=\sum_{n=0}^{N M-1} n e^{-j \frac{2 \pi}{N}(k-f) n} \cos \left(\frac{2 \pi}{N M} p n\right)
$$

and

$$
\gamma(k-f)=\sum_{n=0}^{N M-1} n e^{-j \frac{2 \pi}{N}(k-f) n} .
$$

Since $\sum_{n=0}^{N M-1} e^{-j 2 \pi / N(k-f) n}=\sum_{n=0}^{N M-1} e^{-j 2 \pi / N(k-f) n}$ $\cos ((2 \pi) /(N M) p n)=0$, for $p=1,2, \ldots M-1$, and 
$k-f=-2 N, \ldots,-1,1, \ldots 2 N$, and by using the approximate formula (32), we can express $E\left\{\left|\operatorname{ICI}_{j}^{(1)}[k]\right|^{2}\right\}$ for $0<f_{D} T_{s}<1 / 2 \pi N M$ by

$$
\begin{aligned}
E\left\{\left|\operatorname{ICI}_{j}^{(1)}[k]\right|^{2}\right\} & \simeq 2 N_{h}\left(\frac{\pi\left(f_{D} T_{s}\right) \sigma_{d} \sigma_{x_{i}}}{N M}\right)^{2} \sum_{f=0, f \neq k}^{N-1}\left\{|\gamma(k-f)|^{2}\right. \\
& +\frac{4}{M} \Re\left\{\gamma^{*}(k-f) \sum_{p=1}^{M-1} r_{j, j}[p] \beta(p, k-f)\right\} \\
& \left.+\frac{4}{M^{2}}\left|\sum_{p=1}^{M-1} r_{j, j}[p] \beta(p, k-f)\right|^{2}\right\}
\end{aligned}
$$

where $\Re\{\cdot\}$ denotes the real part. We prove in the Appendix that the real part of $\beta(p, k-f)$ is equal to $-N M / 2$. Based on this result, we can rewrite the ICI average power as

$$
\begin{aligned}
E\left\{\left|\operatorname{ICI}_{j}^{(1)}[k]\right|^{2}\right\} & \simeq 2 N_{h}\left(\frac{\pi\left(f_{D} T_{s}\right) \sigma_{d} \sigma_{x_{i}}}{N M}\right)^{2} \sum_{f=0, f \neq k}^{N-1}\left\{|\gamma(k-f)|^{2}\right. \\
& +\frac{4}{M} \Re\left\{\gamma^{*}(k-f)\left[\Gamma+\sum_{p=1}^{M-1} r_{j, j}[p] \Im\{\beta(p, k-f)\}\right]\right\} \\
& \left.+\frac{4}{M^{2}}\left|\Gamma+\sum_{p=1}^{M-1} r_{j, j}[p] \Im\{\beta(p, k-f)\}\right|^{2}\right\}
\end{aligned}
$$

where $\Im\{\cdot\}$ is the imaginary part and $\Gamma$ can be obtained by (51) as

$$
\Gamma= \begin{cases}\frac{-(M-1) M^{2} N}{4}, & \text { all-one codeword } \\ \frac{M^{2} N}{4}, & \text { otherwise. }\end{cases}
$$

The quantity $r_{j, j}[p]$ can be interpreted as the autocorrelation of codeword $j$. Since $\Im\{\beta(p, k-f)\}$ is a monotonically decreasing or increasing function of $p$ for given $k-f$ as shown in the Appendix, we can characterize the ICI values qualitatively by $r_{j, j}[p]$ based on (54). If $r_{j, j}[p], 1 \leq p \leq M-1$, has a sufficient number of sign changes, the $\sum_{p=1}^{\bar{M}-1} r_{j, j}[p] \Im\{\beta(p, k-f)\}$ term in (54) is likely to be cancelled out after the summation over all $p$, which leads to a smaller ICI value. Since $r_{j, j}[p]=$ $\sum_{q=0}^{M-1-p} w_{j}[p+q] w_{j}[q]$, for $1 \leq p \leq M-1$, there is a relation between the number of sign changes in the codewords and the number of sign changes in $r[p]$. For example, let $M=8$. For the all-one codeword, $r_{1,1}[p]$ is $(7654321)$ that has no sign change. For the second codeword, $(1-11-11-11-1)$ with seven sign changes, $r_{2,2}[p]$ is $(-76-54-32-1)$ that has six sign changes. Finally, for the seventh codeword (11$1-1-1-111)$, the associated $r_{7,7}[p]$ is $(3-2-3-4-121)$ and they both have two sign changes.

With the previous observation, we can adopt the following rule of thumb for codeword selection in a PMU-OFDM system: "To give a higher priority to codewords that have a higher

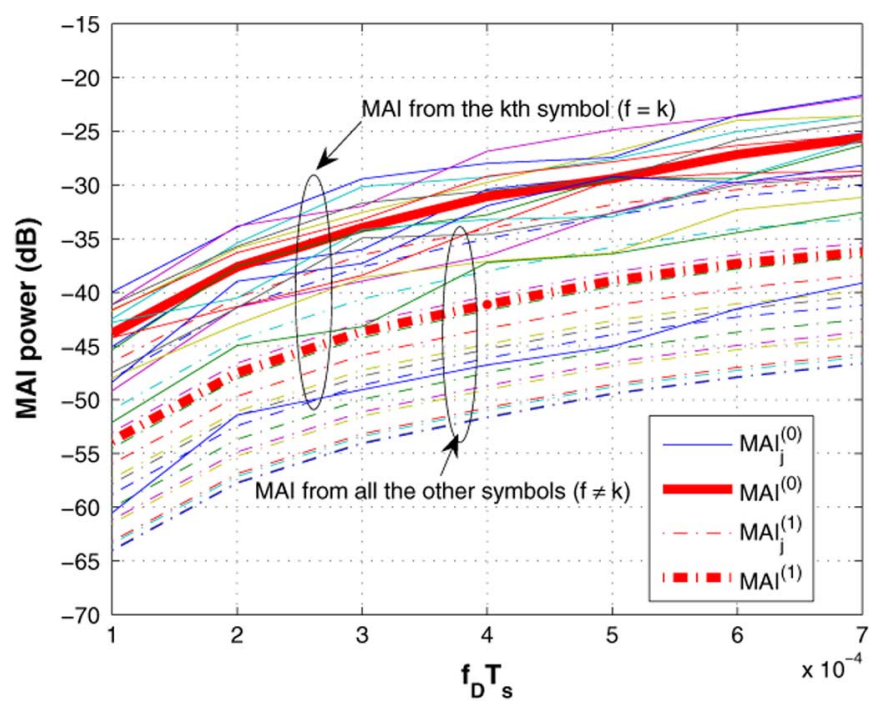

Fig. 3 Dominating and the residual MAI curves as a function of the normalized Doppler frequency.

number of sign-changes." This result is similar to PMU-OFDM in the presence of a pure CFO environment [21]. Simulation results about the code priority scheme will be shown in Section IV.

\section{Simulation Results}

Simulation results are presented in this section to corroborate the theoretical results derived in previous sections. In the simulation, we choose carrier frequency $f_{c}=4 \mathrm{GHz}$ and sampling frequency $F_{s}=1 / T_{s}=2 \mathrm{MHz}$. The maximum Doppler frequency is related to the mobile speed via $f_{D}=f_{c}(V / c)$, where $c$ is the speed of light and $V$ is the mobile speed. Channel coefficients are allowed to change during one OFDM block, and they have the Rayleigh distribution with a unit variance at the same path but different time indices. The classical Jakes Doppler spectrum [11] is adopted for the time-varying fading channel, and the binary phase shift keying (BPSK) modulation scheme is considered. The simulation is conducted by the Monte Carlo method.

Example 1: Suppression of Dominating MAI: In this example, we show that $\mathrm{MAI}_{j \leftarrow i}^{(0)}[k]$ is the dominating MAI as derived in Section III, and then, we investigate its suppression by using even or odd Hadamard-Walsh codewords. The simulation parameters are $N=4, M=16$, and $N_{h}=1$. The average MAI for user $j$ from all other users, denoted by $\overline{\operatorname{MAI}}_{j}^{(0)}$, is calculated as follows. For the $k$ th symbol of any target user, we accumulate the MAI contributed from all the $k$ th symbols of other users. This procedure is repeated for all symbols, and the MAI power is then averaged for $0 \leq k \leq N-1$; i.e., $\overline{\operatorname{MAI}}_{j}^{(0)}=1 / N \sum_{k=0}^{N-1}\left|\sum_{i=1, i \neq j}^{T} \operatorname{MAI}_{j \leftarrow i}^{(0)}[k]\right|^{2}$. This is conducted for more than 400000 symbols in the simulation. Similarly, the total MAI from all other symbols $(f \neq k)$ of other users to user $j$, denoted by $\overline{\mathrm{MAI}}_{j}^{(1)}$, is obtained by averaging the value $(1 / N) \sum_{k=0}^{N-1}\left|\sum_{i=1, i \neq j}^{T} \operatorname{MAI}_{j \leftarrow i}^{(1)}[k]\right|^{2}$. The total MAI is plotted as a function of the maximum normalized Doppler frequency in Fig. 3, where $\overline{\mathrm{MAI}}_{j}^{(0)}$ and $\overline{\mathrm{MAI}}_{j}^{(1)}$ of all 16 users are shown by 16 solid curves and 16 dashed 


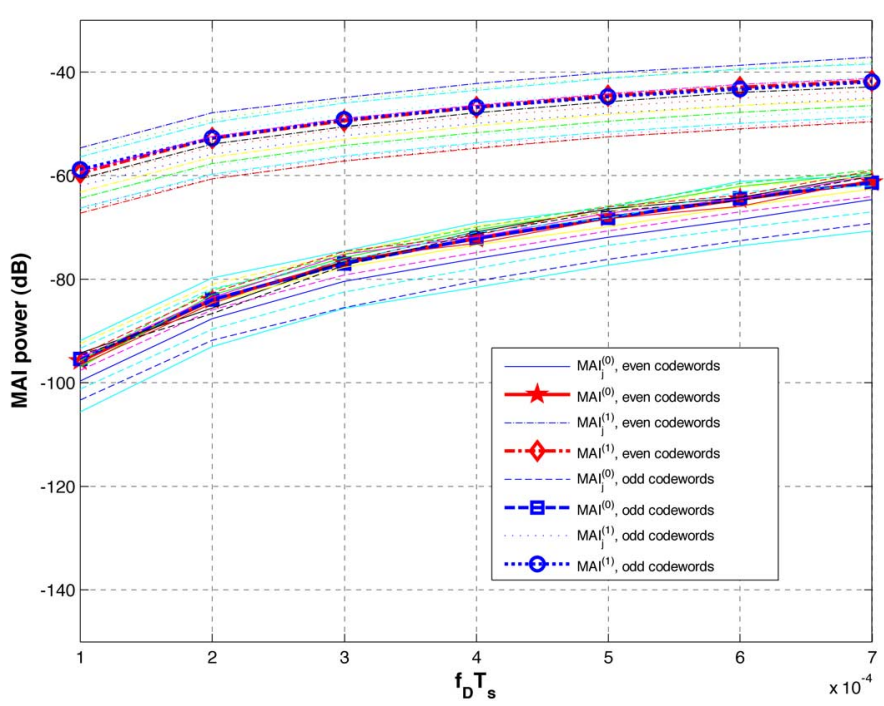

Fig. 4 Dominating and residual MAI versus the normalized Doppler frequency when only $M / 2$ even or odd Hadamard-Walsh codewords are used.

curves, respectively. The solid bold curve in Fig. 3, denoted by $\overline{\mathrm{MAI}}^{(0)}$, is the average value of 16 solid curves and obtained by $(1 / T) \sum_{j=1}^{T} \overline{\mathrm{MAI}}_{j}^{(0)}$. Similarly, the dashed bold curve, denoted by $\overline{\mathrm{MAI}}^{(1)}$, is the average value of 16 dashed curves and obtained by $1 / T \sum_{j=1}^{T} \overline{\mathrm{MAI}}_{j}^{(0)}$. We see from this figure that the average $\overline{\mathrm{MAI}}^{(0)}$ is about $10 \mathrm{~dB}$ more than the average $\overline{\mathrm{MAI}}^{(1)}$. Thus, we can view $\overline{\mathrm{MAI}}^{(0)}$ and $\overline{\mathrm{MAI}}^{(1)}$ as the dominating MAI and the residual MAI, respectively.

Now, we consider a case where only $M / 2=8$ even (or only $M / 2=8$ odd) Hadamard-Walsh codewords are used. The MAI performance is shown for both even and odd codewords in Fig. 4. By comparing results in this figure and Fig. 3, we see that the dominating MAI is reduced by $35-58 \mathrm{~dB}$. In contrast, the residual MAI is only decreased by $5-6 \mathrm{~dB}$ due to the decreased user number from 16 to 8 . The results confirm that the dominating MAI can be greatly reduced using only $M / 2$ even or $M / 2$ odd codewords so that the total MAI, which is equal to $\overline{\mathrm{MAI}}^{(0)}+\overline{\mathrm{MAI}}^{(1)}$, is reduced considerably.

Example 2: Doppler ICI: In this example, we consider the ICI effect due to the Doppler spread. As mentioned previously, we focus on $\mathrm{ICI}_{j}^{(1)}$ for user $j$, whose average power is calculated as follows. For the $k$ th symbol of the target user, we accumulate the ICI from all other symbols $f \neq k$ of the same user. The average ICI power is then averaged for $0 \leq k \leq N-1$; namely, $\overline{\mathrm{ICI}}_{j}^{(1)}=(1 / N) \sum_{k=0}^{N-1}\left|\mathrm{ICI}_{j}^{(1)}[k]\right|^{2}$. Fig. 5 shows the average ICI power for each individual Hadamard-Walsh codeword used in PMU-OFDM when $N=64, M=16$, and $N_{h}=4$. The maximum Doppler frequency was chosen to be $10^{-4}$, which corresponds to the user speed of $54 \mathrm{~km} / \mathrm{h}$. We see that different users experience a different amount of ICI. Especially, the user that employs the all-one codeword suffers more ICI than all others by $15-40 \mathrm{~dB}$. Intuitively, if a codeword has more sign changes, the main lobes of interfering subcarriers may cancel each other so that the ICI power is decreased. This is similar to the self-ICI cancellation technique used to mitigate ICI in OFDM [23]. Since the all-one codeword belongs to the set of

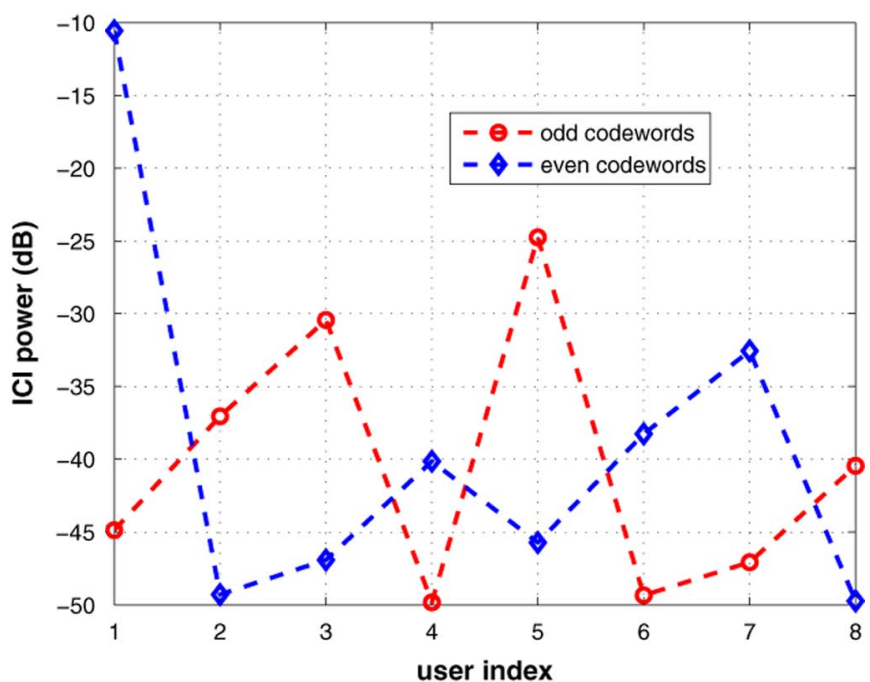

Fig. $5 \mathrm{ICI}^{(1)}$ power as a function of user index for even and odd codewords with $f_{D} T_{s}=1 \times 10^{-4}$.

even Hadamard-Walsh codewords, we recommend to choose the set of odd Hadamard-Walsh codewords first, since they have the approximately MAI-free property and a lower average ICI power at the same time.

In the next several examples, we compare the performance of the PMU-OFDM and the OFDMA systems. For fair comparison, we keep the size of IDFT/DFT of both systems the same, i.e., NM, and consider both fully loaded and half-loaded situations. For a fully loaded OFDMA system, subchannels indexed by $(j-1)+k M, 1 \leq j \leq M$ and $0 \leq k \leq N-1$ are assigned to user $j$. In other words, each user occupies $N$ subchannels which are maximally separated. To simulate the half-loaded PMU-OFDM system, the set of odd (or even) Hadamard-Walsh codewords is used. For the half-loaded OFDMA system, the $j$ th user is assigned subchannels with indices $2(j-1)+k M, 0 \leq$ $k \leq N-1$, and $1 \leq j \leq M / 2$. The remaining subchannels are used as the guard band.

Example 3: Performance Comparison of PMU-OFDM to OFDMA in the Doppler Environment: In this example, we compare the performance of odd and even Hadamard-Walsh codewords for PMU-OFDM with parameters $N=64, M=16$, and $N_{h}=4$. The ICI power and the MAI power for OFDMA and PMU-OFDM are plotted as functions of the normalized Doppler frequency in Fig. 6, where the average ICI power is the averaged value of eight multiple access users (i.e., $T=8$ ). We see that the second largest amount of interference is the ICI of PMU-OFDM with only even codewords. The reason is that the all-one codeword in the set of even codewords has a high ICI value as compared to the ICI or the MAI of all other users. On the other hand, the ICI value of PMU-OFDM with the set of odd Hadamard-Walsh codewords is only about $4 \mathrm{~dB}$ more than that of OFDMA. Note that subcarriers assigned to a particular user in OFDMA are spread uniformly across the available bandwidth. Hence, ICI results in less impairment in OFDMA than in a single-user OFDM. However, for OFDMA, the MAI power is significantly higher than that of PMU-OFDM. As shown in Fig. 6, we observe that the MAI value of PMU-OFDM with either even or odd Hadamard-Walsh codewords is about 


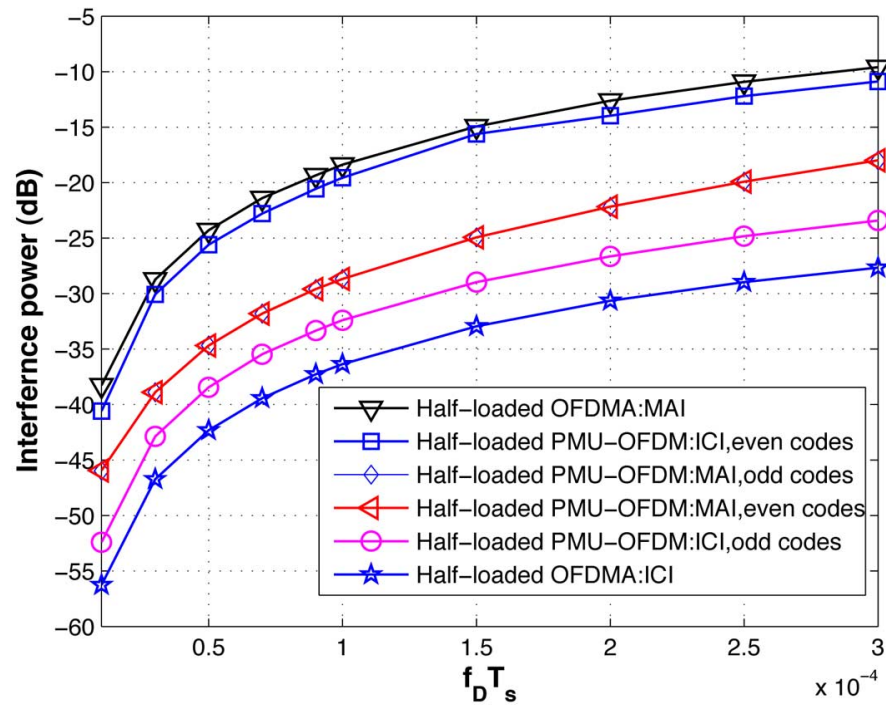

Fig. 6 Comparison of the MAI power and the ICI power as a function of the normalized Doppler frequency.

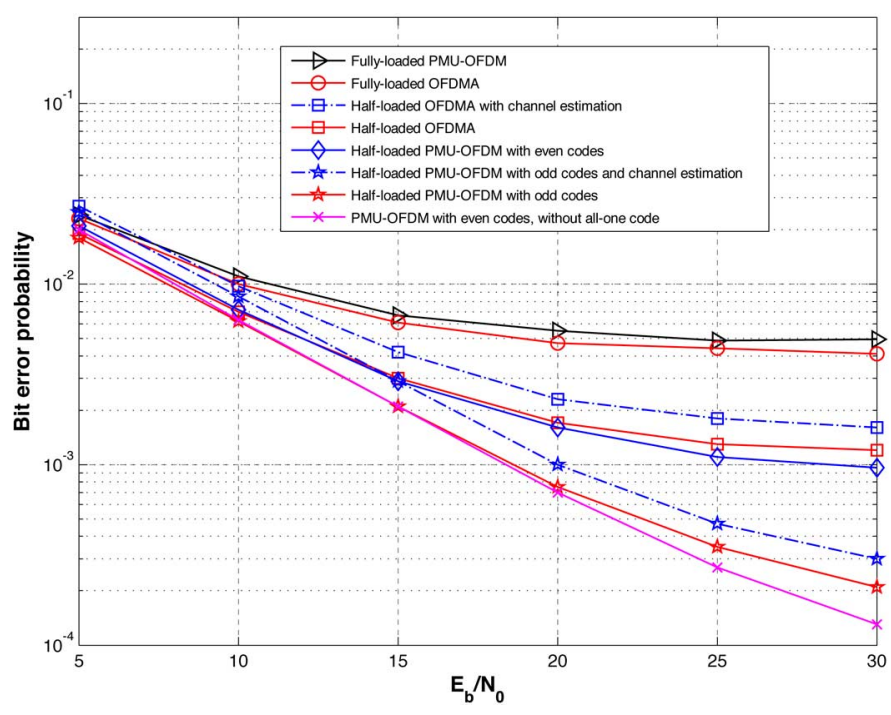

Fig. 7 BEP comparison for PMU-OFDM and OFDMA as a function of the normalized Doppler frequency.

$10 \mathrm{~dB}$ less than that of OFDMA. Thus, we expect PMU-OFDM with only odd codewords to outperform OFDMA in the bit error probability (BEP).

Under the setting of $N=64, M=16, N_{h}=4$, and $E_{b} / N_{0}=30 \mathrm{~dB}$, simulation results on the BEP are shown in Fig. 7, where the BER performance is plotted as a function of the Doppler frequency for six systems: fully loaded PMU-OFDM, fully loaded OFDMA, half-loaded PMU-OFDM with even codewords, half-loaded PMU-OFDM with odd codewords, PMU-OFDM with even codewords, excluding the all-one codeword, and half-loaded OFDMA. In this simulation, we assume that perfect channel knowledge is available at the receiver. An FEQ can be used to compensate the symbol distortion effect, i.e., the detected symbol $\hat{x}_{j}[k]$ is multiplied by $\left(H_{j}[k]\right)^{-1}$. Thus, both systems only suffer from MAI and ICI.

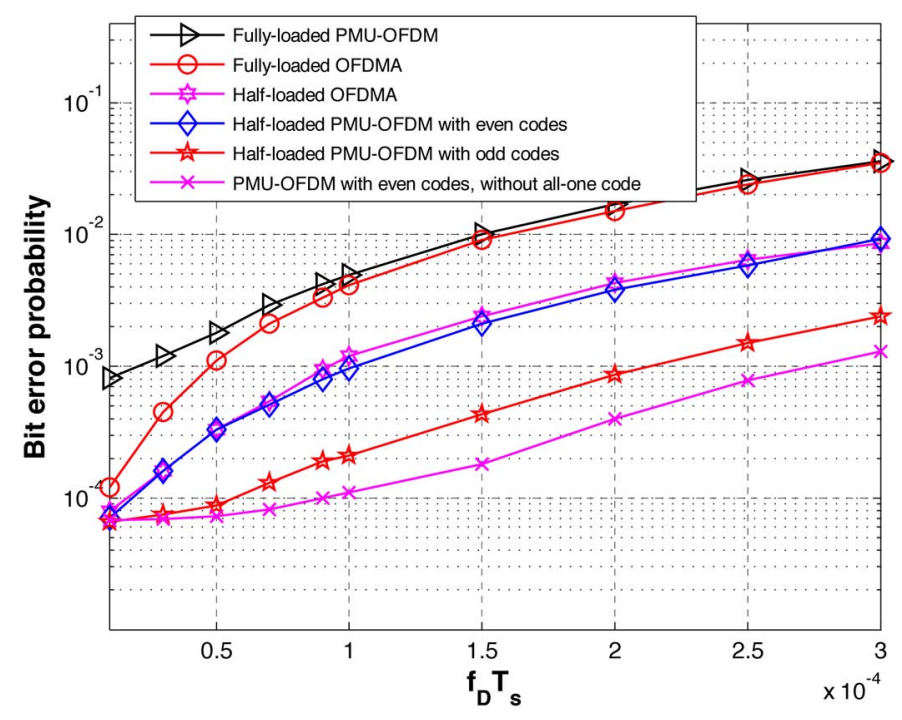

Fig. 8 BEP comparison for PMU-OFDM and OFDMA as a function of the SNR value.

We see that fully loaded PMU-OFDM and fully loaded OFDMA have comparable performance for $f_{D} T_{s} \geq 10^{-4}$. For $f_{D} T_{s}<10^{-4}$ that corresponds to the mobile speed of $54 \mathrm{~km} / \mathrm{h}$ with respect to our chosen parameters, OFDMA outperforms PMU-OFDM since OFDMA is MAI-free if time or frequency asynchronism is negligible. For half-loaded systems, we see that PMU-OFDM with odd codewords yields much lower BEP than PMU-OFDM with even codewords and OFDMA in the Doppler environment with the maximum normalized Doppler frequency ranging from $1 \times 10^{-5}$ to $3 \times 10^{-4}$, corresponding to a mobile speed between 5.4 and $162 \mathrm{~km} / \mathrm{h}$. The poorer performance of half-loaded PMU-OFDM with even codewords is due to high ICI of the user with the all-one codeword. Thus, if we exclude the all-one codeword, PMU-OFDM with seven even codewords outperforms all other systems as shown in Fig. 7.

Now, let us consider nonperfect channel estimation in a time-varying channel. Fig. 8 compares the BEPs for the six scenarios given in Fig. 7 and two more scenarios with channel estimation. That is, we no longer assume perfect knowledge of channel at the receiver. The channel estimation was performed using (43) and (44). We used eight pilots in every OFDM block. The BEP results of all eight scenarios are plotted as a function of the SNR value $E_{b} / N_{0}$, with the maximum normalized Doppler frequency fixed at $10^{-4}$. For fully loaded cases, the BEP curves of PMU-OFDM and OFDMA are close to each other. For half-loaded cases, the BEP curves of PMU-OFDM with even codewords and OFDMA are also close with each other, while PMU-OFDM with odd codewords outperforms the previous four cases considerably. PMU-OFDM with seven even codewords (excluding the all-one codeword) perform much better than half-load PMU-OFDM with all eight even codewords due to the high ICI value of the user with all-one codeword. Also, we see from Fig. 8 that the channel estimation penalty is about $1.5-2.0 \mathrm{~dB}$ for $E_{b} / N_{0} \leq 20 \mathrm{~dB}$. The performance gap between half-loaded PMU-OFDM with odd codewords and half-loaded OFDMA, however, remains the same with realistic channel estimation. 
TABLE I

ICI AVERAGe POWER IN DECIBELS FOR 16 USERS

\begin{tabular}{|c|c|c|c|c|c|c|c|c|}
\hline \# sign changes for even codewords & 0 & 2 & 4 & 6 & 8 & 10 & 12 & 14 \\
\hline ICI of even codewords (dB) & -10.63 & -32.46 & -40.22 & -38.36 & -49.40 & -49.89 & -46.94 & -45.84 \\
\hline \# sign changes for odd codewords & 1 & 3 & 5 & 7 & 9 & 11 & 13 & 15 \\
\hline ICI of odd codewords (dB) & -24.80 & -30.54 & -40.51 & -37.10 & -49.56 & -49.85 & -47.23 & -45.03 \\
\hline
\end{tabular}

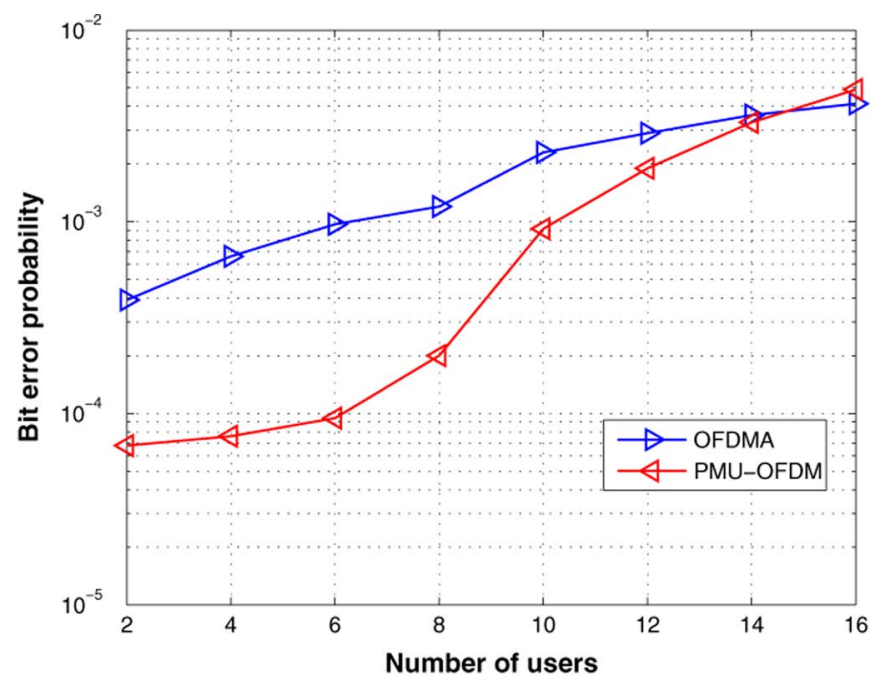

Fig. 9 BEP performance comparison as a function of the user number for PMUOFDM and OFDMA, where the codeword priority scheme is adopted for PMUOFDM.

Example 4: Codeword Priority: It was mentioned in Section III that codewords with a higher number of sign changes in general have a lower amount of ICI. We conduct simulations to verify this statement here. System parameters are chosen to be $N=64, M=16$, and $N_{h}=4$. The maximum normalized Doppler frequency and the SNR value were fixed at $10^{-4}$ and $30 \mathrm{~dB}$, respectively.

The ICI power for 16 codewords is shown in Table I, where the corresponding number of sign changes is also provided. It is a general trend that codewords with more sign changes have less ICI.

Next, we examine how PMU-OFDM and OFDMA perform when the active number of users varies from 1 to $T=M$. The codeword priority scheme for PMU-OFDM is stated later. When the system load is less than $50 \%$, we use codewords with a higher number of sign changes among the $M / 2$ odd codewords. When the system load is more than $50 \%$, we choose all $M / 2$ odd codewords plus an even codeword predetermined to have a low average ICI value based on the results given in Table I. The procedure is repeated until all 16 codewords are selected. We plot BEP as a function of the user number for PMU-OFDM and OFDMA in Fig. 9.

From Fig. 9, we see that PMU-OFDMA significantly outperforms OFDMA in a lightly loaded (50\% or less) system. When the system reaches its full loading, the BEP performance of PMU-OFDM and OFDMA becomes comparable.

\section{CONCLUSION}

The performance of an approximately MAI-free multiaccess OFDM transceiver, called the PMU-OFDM system, in a mobile environment was analyzed in this paper. It was shown that the MAI due to the Doppler spread can be greatly reduced using either $M / 2$ even or odd codewords of $M$ Hadamard-Walsh codewords in the system. We also investigated the ICI performance of PMU-OFDM in the Doppler environment and showed by simulations that, excluding the all-one codeword, the average ICI power of PMU-OFDM is comparable with that of OFDMA. The codeword selection priority scheme for PMU-OFDM was also discussed. Finally, we demonstrated that PMU-OFDM has a significant performance gain over OFDMA for a wide range of system loading in a mobile environment in terms of BEP performance. As the system loading becomes higher, such a performance gain becomes narrower. For a fully loaded system, PMU-OFDM and OFDMA have comparable BEP performance.

\section{APPENDIX}

Our objective is to prove that the real part of $\beta(p, k-f)$ is constant while the imaginary part of $\beta(p, k-f)$ is a monotonic function of $p$ for a given value of $k-f$. Let $k-f=l$, where $l=-(N-1), \ldots,-1,0,1, \ldots, N-1$.

First, we show that the real part is constant. The real part of $\beta(p, l)$ can be written as

$$
\begin{aligned}
\Re\{\beta(p, l)\}= & \sum_{n=0}^{N M-1} n \cos \left(\frac{2 \pi}{N M}(l M) n\right) \cos \left(\frac{2 \pi}{N M} p n\right) \\
= & \frac{1}{2}\left\{\sum_{n=0}^{N M-1} n \cos \left(\frac{2 \pi}{N M}(l M+p) n\right)\right. \\
& \left.+\sum_{n=0}^{N M-1} n \cos \left(\frac{2 \pi}{N M}(l M-p) n\right)\right\} .
\end{aligned}
$$

With (34), we get

$$
\begin{aligned}
& \Re\{\beta(p, l)\}=\frac{1}{2}\left\{\frac{N M \sin \left(2 \pi(l M+p)-\frac{\pi}{N M}(l M+p)\right)}{2 \sin \left(\frac{\pi}{N M}(l M+p)\right)}\right. \\
&\left.-\frac{1-\cos (2 \pi(l M+p))}{4 \sin ^{2}\left(\frac{\pi}{N M}(l M+p)\right)}\right\} \\
&+ \frac{1}{2}\{N M \sin (2 \pi(l M-p) \\
&\left.-\frac{\pi}{N M}(l M-p)\right) 2 \sin \left(\frac{\pi}{N M}(l M-p)\right) \\
&\left.-\frac{1-\cos (2 \pi(l M-p))}{4 \sin ^{2}\left(\frac{\pi}{N M}(l M-p)\right)}\right\} .
\end{aligned}
$$




$$
\begin{cases}\left|\sin \left(\frac{\pi}{N M}(l M+p)\right)\right| \geq\left|\sin \left(\frac{\pi}{N M}(l M-p)\right)\right|, & \text { if } 0 \leq l \leq N / 2 \\ \left|\sin \left(\frac{\pi}{N M}(l M+p)\right)\right| \leq\left|\sin \left(\frac{\pi}{N M}(l M-p)\right)\right|, & \text { if } N / 2 \leq l \leq N-1 .\end{cases}
$$

Since $\sin (2 \pi(l M+p)-(\pi) /(N M)(l M+p))=$ $-\sin ((\pi) /(N M)(l M+p))$ and $\cos (2 \pi(l M+p))=0$ for $p=1,2, \ldots M-1$ and $l=-(N-1), \ldots,(N-1)$, we have $\Re\{\beta(p, l)\}=(-N M) /(4)+(-N M) /(4)=(-N M) /(2)$.

Next, we prove that the imaginary part of $\beta$ is a monotonic function of $p$ given $l=k-f$. The imaginary part of $\beta(p, l)$ can be written as

$$
\begin{aligned}
& \Im\{\beta(p, l)\}= \sum_{n=0}^{N M-1}-n \sin \left(\frac{2 \pi}{N M}(l M) n\right) \cos \left(\frac{2 \pi}{N M} p n\right) \\
&=\frac{-1}{2}\left\{\sum_{n=0}^{N M-1} n \sin \left(\frac{2 \pi}{N M}(l M+p) n\right)\right. \\
&\left.+\sum_{n=0}^{N M-1} n \sin \left(\frac{2 \pi}{N M}(l M-p) n\right)\right\} .
\end{aligned}
$$

Based on the following equality given in [7]:

$$
\sum_{k=1}^{n-1} k \sin (k x)=\frac{\sin (n x)}{4 \sin ^{2}\left(\frac{x}{2}\right)}-\frac{n \cos \left(\frac{2 n-1}{2} x\right)}{2 \sin \left(\frac{x}{2}\right)}, \quad x \neq 0
$$

we can express $\Im\{\beta(p, l)\}$ as

$$
\begin{aligned}
\Im & \{\beta(p, l)\} \\
= & \frac{-1}{2}\left\{\frac{\sin (2 \pi(l M+p))}{4 \sin ^{2}\left(\frac{\pi}{N M}(l M+p)\right)}\right. \\
& \left.\quad-\frac{N M \cos \left(2 \pi(l M+p)-\frac{\pi}{N M}(l M+p)\right)}{2 \sin \left(\frac{\pi}{N M}(l M+p)\right)}\right\} \\
+ & \frac{-1}{2}\left\{\frac{\sin (2 \pi(l M-p))}{4 \sin ^{2}\left(\frac{\pi}{N M}(l M-p)\right)}\right. \\
& \left.\quad-\frac{N M \cos \left(2 \pi(l M-p)-\frac{\pi}{N M}(l M-p)\right)}{2 \sin \left(\frac{\pi}{N M}(l M-p)\right)}\right\} \\
= & \frac{N M}{4} \cot \left(\frac{\pi}{N M}(l M+p)\right)+\frac{N M}{4} \cot \left(\frac{\pi}{N M}(l M-p)\right) .
\end{aligned}
$$

To prove $\Im\{\beta(p, l)\}$ is a monotonic function of $p$ given $l$, we show that its derivative with respect to $p$ is either negative or positive for any $p$ given $l$. By taking the derivative of $\Im\{\beta(p, l)\}$ with respect to $p$, we get

$$
\begin{aligned}
\frac{\partial \Im\{\beta(p, l)\}}{\partial p}=\frac{\pi}{4}\left\{\frac{-1}{\sin ^{2}\left(\frac{\pi}{N M}(l M+p)\right)}\right. \\
\left.+\frac{1}{\sin ^{2}\left(\frac{\pi}{N M}(l M-p)\right)}\right\} .
\end{aligned}
$$

To understand the behavior of $\partial \Im\{\beta(p, l)\} / \partial p$, we have

$$
\begin{aligned}
\sin \left(\frac{\pi}{N M}(l M+p)\right)= & \sin \left(\frac{\pi}{N} l\right) \cos \left(\frac{\pi}{N M} p\right) \\
& +\cos \left(\frac{\pi}{N} l\right) \sin \left(\frac{\pi}{N M} p\right)
\end{aligned}
$$

and

$$
\begin{aligned}
\sin \left(\frac{\pi}{N M}(l M-p)\right)= & \sin \left(\frac{\pi}{N} l\right) \cos \left(\frac{\pi}{N M} p\right) \\
& -\cos \left(\frac{\pi}{N} l\right) \sin \left(\frac{\pi}{N M} p\right)
\end{aligned}
$$

for $1 \leq p \leq M-1$ and $N \geq 2,(\pi) /(N M) p \leq \pi / 2$. Thus, $\cos ((\pi) /(N M) p) \geq 0$ and $\sin ((\pi) /(N M) p) \geq 0$. For all positive values of $l$, i.e., $0 \leq l \leq(N-1), \sin (\pi / N l) \geq 0$, but $\cos (\pi / N l)$ can take either negative or positive values. If $l \leq N / 2, \cos (\pi / N l) \geq 0$. On the other hand, if $l \geq N / 2, \cos (\pi / N l) \leq 0$. Then, we conclude from (61) and (62) that (63), shown at the top of the page, holds. As a result, $\Im\{\beta(p, l)\}$ is an increasing function of $p$ if $0 \leq l \leq N / 2$ and a decreasing function of $p$ if $N / 2 \leq l \leq N-1$. Similarly, for $l \leq 0$, we can prove that $\Im\{\beta(p, l)\}$ is a decreasing function of $p$ if $-N / 2 \leq l \leq 0$ and an increasing function of $p$ if $-(N-1) \leq l \leq-N / 2$.

\section{REFERENCES}

[1] M. Abramowitz and I. A. Stegun, Handbook of Mathematical Functions With Formulas, Graphs, and Mathematical Tables. Washington, DC: United States Department of Commerce, Dec. 1972.

[2] J. A. C. Bingham, "Multicarrier modulation for data transmission: An idea whose time has come," IEEE Commun. Mag., vol. 28, no. 5, pp. 5-14, May 1990

[3] X. Cai and G. B. Giannakis, "Bounding performance and suppressing intercarrier interference in wireless mobile OFDM," IEEE Trans. Commun., vol. 51, no. 12, pp. 2047-2056, Dec. 2003.

[4] L. J. Cimini, Jr., "Analysis and simulation of a digital mobile channel using orthogonal frequency division multiplexing," IEEE Trans. Commun., vol. 33, no. 7, pp. 665-675, Jul. 1995.

[5] R. Negi and J. Cioffi, "Pilot tone selection for channel estimation in a mobile OFDM system," IEEE Trans. Consum. Electron., vol. 44, no. 3, pp. 1122-1128, Aug. 1998.

[6] Y. Mostofi and D. C. Cox, "ICI mitigation for pilot-aided OFDM mobile systems," IEEE Trans. Wireless Commun., vol. 4, no. 2, pp. 765-774, Mar. 2005.

[7] I. S. Gradshteyn, I. M. Ryzhik, and A. Jeffrey, Tables of Integrals, Series, and Products. New York: Academic, 1994.

[8] S. Hara and R. Prasad, "Overview of multicarrier CDMA," IEEE Commun. Mag., vol. 35, no. 12, pp. 126-133, Dec. 1997.

[9] H. F. Harmuth, "Application of Walsh function in communications," IEEE Spectrum, no. 6, pp. 82-91, Nov. 1969.

[10] IEEE Standard for Local and Metropolitan Area Networks, IEEE 802.16a Standard, Apr. 2003.

[11] W. C. Jakes, Microwave Mobile Communications. New York: Wiley, 1974.

[12] I. Koffman and V. Roman, "Broadband wireless access solutions based on OFDM access in IEEE 802.16," IEEE Commun. Mag., vol. 40, no. 4, pp. 96-103, Apr. 2001.

[13] Y. Li, L. G. Cimini, and N. R. Sollenberger, "Robust channel estimation for OFDM systems with rapid dispersive fading channels," IEEE Trans. Commun., vol. 46, no. 7, pp. 902-915, Jul. 1998. 
[14] Y. Li, "Pilot-symbol-aided channel estimation for OFDM in wireless systems," IEEE Trans. Veh. Technol., vol. 49, no. 4, pp. 1207-1215, Jul. 2000.

[15] J.-P. M. G. Linartz and A. Gorokhov, "New equalization approach for OFDM over dispersive and rapidly time-varying channel," in Proc. PIMRC Conf., London, U.K., Sep. 2000, pp. 710-713.

[16] J. G. Proakis, Digital Communications. , NY: McGraw-Hill, 2000.

[17] P. Schniter, "Low-complexity equalization of OFDM in doubly selective channels," IEEE Trans. Signal Process., vol. 52, no. 4, pp. 1002-1011, Apr. 2004.

[18] K. A. Seation and J. Armstrong, "Polynomial cancellation coding and finite differences," IEEE Trans. Inf. Theory, vol. 46, no. 1, pp. 311-313, Jan. 2000.

[19] S. H. Tsai, Y. P. Lin, and C.-C. J. Kuo, "An approximately MAI-free multiaccess OFDM system in carrier frequency offset environment," IEEE Trans. Signal Process., vol. 53, no. 11, pp. 4339-4353, Nov. 2005.

[20] S. H. Tsai, Y. P. Lin, and C.-C. J. Kuo, "A precoded multiuser OFDM (PMU-OFDM) transciever for time asynchronous systems," in Proc. IEEE Global Telecommun. Conf., Dec. 2005, vol. 4, pp. 2214-2218.

[21] S. H. Tsai, Y. P. Lin, and C.-C. J. Kuo, "Code priority of Multiuser OFDM systems in frequency asynchronous environment," in Proc. IEEE 62nd Semiannu. Veh. Technol. Conf., Dallas, TX, Sep. 25-28, 2005, pp. 1103-1107.

[22] S. Verdu, Multiuser Detection. Cambridge, U.K.: Cambridge Univ. Press, 1998.

[23] Y. Zhao and S. G. Haggman, "Intercarrier interference self-cancellation scheme for OFDM mobile communication systems," IEEE Trans. Commun., vol. 49, no. 7, pp. 1185-1191, Jul. 2001.

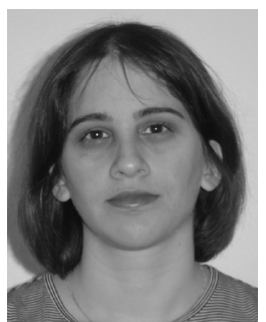

Layla Tadjpour (S'07) received the B.S. degree in electrical engineering from the Iran University of Science and Technology, Tehran, Iran, in 1996 and the M.S. degree in electrical engineering from the University of California, Los Angeles, in 1999. Currently, she is working towards the Ph.D. degree at the Ming-Hsieh Department of Electrical Engineering, University of Southern California, Los Angeles.

From 1999 to 2005, she was with the Jet Propulsion Laboratory, Pasadena, CA. Her current research interests are in the area of signal processing for communications, multicarrier systems, and mobile communications.

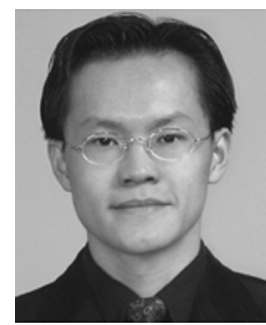

Shang-Ho Tsai (S'04-M'06) was born in Kaohsiung, Taiwan, in 1973. He received the B.S. degree in electrical engineering from the Tamkang University, Tamsui, Taiwan, in 1995, the M.S. degree in electrical and control engineering from the National Chiao-Tung University, Hsinchu, Taiwan, in 1999, and the Ph.D. degree in electrical engineering from the University of Southern California, Los Angeles, in 2005.

From 1999 to 2002, he was with the Silicon Integrated Systems Corporation (SiS), Taiwan, where he participated in the very large scale integration (VLSI) design for DMT-ADSL systems. From 2005 to 2007, he was with MediaTek, Inc. (MTK), Taiwan, where he participated in the VLSI design for MIMO-OFDM systems. In 2007, he joined the Department of Electrical and Control Engineering, National ChiaoTung University. His research interests include signal processing for communications, particularly in the areas of multicarrier systems and space-time processing. He is also interested in VLSI design for those topics.

Dr. Tsai was awarded a government scholarship for overseas study from the Ministry of Education, Taiwan, in 2002-2005.

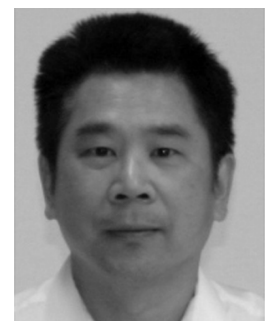

C.-C. Jay Kuo (F'99) received the B.S. degree from the National Taiwan University, Taipei, Taiwan, in 1980 and the M.S. and Ph.D. degrees from the Massachusetts Institute of Technology, Cambridge, in 1985 and 1987, respectively, all in electrical engineering.

He was Computational and Applied Mathematics (CAM) Research Assistant Professor at the Department of Mathematics, University of California, Los Angeles, from October 1987 to December 1988 Since January 1989, he has been with the University of Southern California, Los Angeles, where currently, he is a Professor of Electrical Engineering, Computer Science and Mathematics, and the Director of the Signal and Image Processing Institute. His research interests are in the areas of digital signal and image processing, multimedia compression, communication and networking technologies. He is the coauthor of about 120 journal papers, 680 conference papers, and seven books.

Dr. Kuo is a Fellow of The International Society for Optical Engineering (SPIE). He is the Editor-in-Chief for the Journal of Visual Communication and Image Representation, and Editor for the Journal of Information Science and Engineering, LNCS Transactions on Data Hiding and Multimedia Security, and the EURASIP Journal of Applied Signal Processing. He received the National Science Foundation Young Investigator Award (NYI) and Presidential Faculty Fellow (PFF) Award in 1992 and 1993, respectively. 